\title{
Video Article \\ Measuring Spray Droplet Size from Agricultural Nozzles Using Laser Diffraction
}

\author{
Bradley K. Fritz ${ }^{1}$, W. Clint Hoffmann ${ }^{1}$ \\ ${ }^{1}$ Aerial Application Technology Research Unit, USDA ARS \\ Correspondence to: Bradley K. Fritz at bradfritz@me.com \\ URL: https://www.jove.com/video/54533 \\ DOI: doi:10.3791/54533
}

Keywords: Engineering, Issue 115, Agricultural application, droplet sizing, spray nozzles, aerial application, ground application, droplet measurement, physics

Date Published: 9/16/2016

Citation: Fritz, B.K., Hoffmann, W.C. Measuring Spray Droplet Size from Agricultural Nozzles Using Laser Diffraction. J. Vis. Exp. (115), e54533, doi:10.3791/54533 (2016).

\section{Abstract}

When making an application of any crop protection material such as an herbicide or pesticide, the applicator uses a variety of skills and information to make an application so that the material reaches the target site (i.e., plant). Information critical in this process is the droplet size that a particular spray nozzle, spray pressure, and spray solution combination generates, as droplet size greatly influences product efficacy and how the spray moves through the environment. Researchers and product manufacturers commonly use laser diffraction equipment to measure the spray droplet size in laboratory wind tunnels. The work presented here describes methods used in making spray droplet size measurements with laser diffraction equipment for both ground and aerial application scenarios that can be used to ensure inter- and intra-laboratory precision while minimizing sampling bias associated with laser diffraction systems. Maintaining critical measurement distances and concurrent airflow throughout the testing process is key to this precision. Real time data quality analysis is also critical to preventing excess variation in the data or extraneous inclusion of erroneous data. Some limitations of this method include atypical spray nozzles, spray solutions or application conditions that result in spray streams that do not fully atomize within the measurement distances discussed. Successful adaption of this method can provide a highly efficient method for evaluation of the performance of agrochemical spray application nozzles under a variety of operational settings. Also discussed are potential experimental design considerations that can be included to enhance functionality of the data collected.

\section{Video Link}

The video component of this article can be found at https://www.jove.com/video/54533/

\section{Introduction}

When making any agrochemical spray application, the primary concerns are ensuring maximum biological efficacy while minimizing any offtarget movement and associated adverse environmental impact or other non-target biological harm. One of the principal factors to consider when setting up any sprayer, prior to an application, is droplet size, which has long been recognized as one of the primary parameters influencing overall spray deposition, efficacy, and drift. While there are a number of other factors that potentially impact spray deposition and drift, droplet size is one of the easiest to change to fit the needs of a given application scenario. Droplet size from any agricultural spray nozzle is influenced by a number of factors including, but not limited to, nozzle type, nozzle orifice size, spray pressure and spray solution physical properties. With aerial applications, the additional influence of air shear resulting from the airspeed of the aircraft and the nozzle's orientation relative to that airshear, causes secondary breakup of the sprays leaving the nozzles ${ }^{1}$. With all of these factors, applicators are faced with the difficult task of making proper nozzle selection and operational setup decisions that insure that all pesticide products labels are met and that the resulting spray droplet size is such that on-target deposition and biological efficacy are maintained while minimizing off-target movement. The goal of this method is to provide clear, concise information on the droplet size resulting from the various combinations influencing factors to support an applicator's operational decisions.

While there are a number of instruments available for measuring droplet size from sprays, measurements from agrochemical spray nozzles are typically either laser diffraction, imagery, or phase doppler based ${ }^{2}$. The imagery and phase doppler based methods are single particle counter methods, meaning that smaller areas within the spray cloud are focused on, with individual particles being measured ${ }^{3}$. Whereas laser diffraction methods take an ensemble measurement, meaning the distribution of a group of particles is rapidly measured ${ }^{3}$. While these methods differ in principle, with proper setup and use, comparable results can be obtained ${ }^{4}$. Laser diffraction methods have been widely adopted by the agricultural application community due to the ease of use, ability to rapidly measurement high number density sprays and the large dynamic measurement range. As an ensemble measurement is made, a single traverse of a spray plume through the line of measurement is all that is required for a composite droplet size of the entire spray. This allows for efficient evaluations of droplet size from a large number of spray nozzles and operational parameter combinations. By comparison, the single particle counter methods necessarily focus on much smaller areas within a spray cloud in order to capture individual particles, meaning that multiple measurement locations must be evaluated and combined to return a composite result. This requires significantly more time, effort and spray solution to evaluate a single spray plume than laser diffraction based methods. The increased spray volume required can present a significant problem if actual pesticide products are being tested as a result of increased costs of the material used and the disposal costs. However, the single particle counter methods offer the advantage of providing a 
temporal sample, in that they measure the number of droplets per unit time passing through a sample volume, whereas laser diffraction provides a spatial sample as the measurement is proportional the number of droplets within a given volume ${ }^{5}$. Were all droplet velocities within a given spray the same, the methods would provide identical results. However, for most spray systems the droplet velocities are correlated to droplet size, resulting in a bias with spatial sampling methods ${ }^{6}$.

Overcoming this spatial bias from laser diffraction measurements through appropriate testing methodology is a critical part of evaluating spray droplet size from agricultural spray nozzles ${ }^{4}$. The spatial bias is reduced when testing nozzles in a concurrent airstream of $13 \mathrm{~m} / \mathrm{sec}$ and with the measurement location located an appropriate distance from the nozzle, as the combination of these two parameters results in homogeneous droplet velocities throughout the spray cloud ${ }^{4}$. Further, the spatial bias is small ( $5 \%$ or less) for aerial nozzle testing due to the high concurrent airspeeds evaluated ${ }^{7,8}$. To determine the optimal test method to reduce the spatial bias with our current low and high speed wind tunnel facilities, the series of reference nozzles used to determine agricultural spray size classifications ${ }^{9}$ were evaluated for droplet size using both laser diffraction and imaging methods ${ }^{10}$. Sizing evaluations were conducted under multiple combinations of concurrent air velocity and measurement distance (distance from the nozzle exit to the point of measurement), representative of the operational range of the existing facilities. Laser diffraction measurements were compared to imagery results to determine the potential spatial bias and the optimal combination of measurement distance and concurrent airspeed was selected as the standard operational procedure. A measurement distance of $30.5 \mathrm{~cm}$ and a concurrent airspeed of $6.7 \mathrm{~m} / \mathrm{sec}$ for evaluation of ground spray nozzles in the low speed wind tunnel reduced spatial bias to $5 \%$ or less ${ }^{10}$. Spatial biases of $3 \%$ or less were obtained for aerial nozzle evaluations in the high speed tunnel, for all airspeeds tested, with a measurement distance of $45.7 \mathrm{~cm}^{10}$. Using these standard methods, the authors were also able to demonstrate that lab to lab variability could be minimized, providing for consistent interlaboratory droplet size data ${ }^{11}$.

All droplet size testing demonstrated as part of this work was conducted at the USDA-ARS-Aerial Application Technology Research Unit's spray atomization research facility. A laser diffraction system was positioned downstream of the nozzle at the distances specified in the Protocol section. For ground nozzle testing, the laser diffraction system was configured, following the manufacturer's instructions, to have a dynamic size range of $18-3,500 \mu \mathrm{m}$ across 31 bins $^{12}$. Likewise for the aerial nozzle testing the system was configured with a dynamic size range of 9 to $1,750 \mu \mathrm{m}$, also across 31 bins $^{12}$. Aerial based spray nozzle evaluations were conducted in high speed air to simulate aerial application conditions. Ground sprayer nozzles were tested in a larger wind tunnel section with a single concurrent airspeed to minimize the spatial bias from laser diffraction. Nozzles being tested were positioned upstream of the laser diffraction system at the distances given in the Protocol section. Nozzles were mounted on a linear traverse allowing for the spray plume to be traversed vertically through the measurement zone during a given measurement cycle. The Protocol for ground nozzle testing describes an experiment examining three typical nozzles at two spray pressures while the aerial nozzle testing describes an experiment examining two typical spray nozzles at two spray pressures and three airspeeds. Both testing scenarios use an "active blank" spray solution, rather than water only, to mimic the effects of real world spray solutions.

\section{Protocol}

\section{Preliminary Setup and Alignment}

1. Prior to any testing, align the laser diffraction system components following the guidelines provided by the manufacturer to ensure proper system functionality and data quality.

2. Follow proper safety precautions associated with the use of a Class IIla laser avoiding direct eye exposure. Use proper Personal Protection Equipment if active ingredient chemical spray solutions are being used.

\section{Ground Nozzle Droplet Sizing}

1. Prepare the "active blank" by adding $47.5 \mathrm{ml}$ (reflects a mix rate of $0.25 \% \mathrm{v} / \mathrm{v}$ ) of a $90 \%$ non-ionic surfactant to $19 \mathrm{~L}$ of water and mixing well using a stir rod in a cordless drill. Depending on the amount of testing to be done, larger volumes of active blank may be required.

2. Pour the "active blank" spray mixture into the stainless steel pressure tanks, seal the tank and attach the input air pressure hose and the outgoing liquid hose feeding the spray nozzle.

3. Confirm that the distance between the nozzle outlet and the measurement zone is $30.5 \mathrm{~cm}$ (12 in) using a tape measure. If it is, continue. If not, adjust by moving either the laser diffraction system or the nozzle.

4. Install a standard 110 degree flat fan nozzle with a \#05 orifice (noted as an XRC11005 nozzle) in the nozzle body attached to the traverse system. Adjust the nozzle orientation such that the long axis of the flat fan nozzle is oriented vertically in the tunnel but either rotating the nozzle within the mounting ring on the check valve or by changing the position of the check valve if the nozzle cannot be rotated to the correct position.

5. Turn on the wind tunnel and set the airspeed to $6.7 \mathrm{~m} / \mathrm{sec}$ by adjusting the fan speed and confirming the airspeed in the tunnel using a hot wire anemometer.

6. Set the spray pressure to $276 \mathrm{kPa}(40 \mathrm{psi})$ by adjusting the incoming air pressure using an inline pressure regulator. Confirm the pressure using an electronic pressure gauge installed immediately upstream of the spray nozzle.

7. Position the nozzle at the top of the tunnel by activating and running the linear traverse to the top-most position prior to initiating the measurement process.

8. Ensure that all experimental parameters (nozzle, pressure, solution, etc.) are properly recorded in the laser diffraction system data recording software by confirming that the parameters recorded on the User Parameters interface window match the testing conditions. NOTE: This data parameter recording screen may vary by laser diffraction instrument.

9. Initiate a reference measurement by selecting the Reference Measurement icon in the operating software to account for any dust or background particles.

10. Initiate start of the measurement cycle. Depending on the laser diffraction system being used, a few seconds is typically required to focus the sensor prior to initiating the measurement process. 
11. Once the system indicates it is ready to start the measurement process, activate the spray by opening the liquid feed valve on the pressure tank. Once the spray is started, lower the nozzle through the laser beam using the traverse mechanism until the entire spray plume has passed through the measurement zone. Deactivate the spray by closing the liquid feed valve.

NOTE: On the laser diffraction system used by the authors, the actual measurement process does not initiate until the spray passing through the measurement zone achieves an optical concentration of $0.5 \%$, and continues until an elapsed time of 10-12 sec has elapsed. These settings will vary by laser diffraction system and user settings.

12. Repeat steps $2.7-2.11$ for a minimum of 3 replicates. Determine if additional replicates are required by computing the mean and standard deviation for the $D_{\mathrm{V}_{0.1},}, D_{\mathrm{v} 0.5}$, and $D_{\mathrm{V} 0.9}$ of the three replicates and ensuring that standard deviation is $10 \%$, or less, of the mean. Perform additional replicates as needed to meet the criteria.

13. Set the spray pressure to $414 \mathrm{kPa}(60 \mathrm{psi})$ and repeat steps $2.7-2.12$.

14. Repeat steps $2.6-2.12$ for each additional nozzle and pressure combination of interest.

15. Export and save droplet size data using the method provided within the operating software.

\section{Aerial Nozzle Droplet Sizing}

1. Prepare the "active blank" by adding $47.5 \mathrm{ml}$ of a $90 \%$ non-ionic surfactant to $19 \mathrm{~L}$ of water and mixing well using a stir rod in a cordless drill. NOTE: depending on the amount of testing to be done, larger volumes of active blank may be required.

2. Pour the "active blank" spray mixture into the stainless steel pressure tanks, seal the tank and attach the input air pressure hose and the outgoing liquid hose feeding the spray nozzle.

3. Confirm that the distance between the nozzle outlet and the measurement zone is $45.7 \mathrm{~cm}$ (18 in) using a tape measure. If it is, continue. If not, adjust by moving the laser diffraction system the required distance from the nozzle.

4. Install a standard 20 degree flat fan nozzle with a \#15 orifice (noted as a 2015 nozzle) in a check valve and nozzle body onto the boom traverse section at the wind tunnel outlet. Ensure that the nozzle is correctly positioned with the nozzle body oriented horizontally and parallel to the airstream.

5. Turn on the wind tunnel blower and set the airspeed at the tunnel outlet to $53.6 \mathrm{~m} / \mathrm{sec}(120 \mathrm{mph})$ and confirm speed using pitot tube attached to an airspeed indicator.

6. Set the spray pressure to $207 \mathrm{kPa}(30 \mathrm{psi})$ by adjusting the incoming air pressure using an inline pressure regulator.

7. Position the nozzle at the top position of the traverse prior to initiating the measurement process.

8. Ensure that all experimental parameters (nozzle, pressure, solution, etc.) are properly recorded in the laser diffraction system data recording software by confirming that the parameters recorded on the User Parameters interface window match the testing conditions. NOTE: This data parameter recording screen may vary by laser diffraction instrument.

9. Initiate a reference measurement by selecting the Reference Measurement icon in the operating software to account for any dust or background particles.

10. Initiate start of the measurement cycle. Depending on the laser diffraction system being used, a few seconds is typically required to focus the sensor prior to initiating the measurement process.

11. Once the system indicates it is ready to start the measurement process, activate the spray by opening the liquid feed valve on the pressure tank. Once the spray is started, lower the nozzle through the laser beam using the traverse mechanism until the entire spray plume has passed through the measurement zone. Deactivate the spray by closing the liquid feed valve.

NOTE: On the laser diffraction system used by the authors, the actual measurement process does not initiate until the spray passing through the measurement zone achieves an optical concentration of $0.5 \%$, and continues until an elapsed time of 5-7 sec has elapsed. These settings will vary by laser diffraction system and user settings.

12. Repeat steps $3.7-3.11$ for a minimum of 3 replicates. Determine if additional replicates are required by computing the mean and standard deviation for the $D_{v_{0} .1}, D_{v_{0.5}}$, and $D_{v_{0.9}}$ of the three replicates and ensuring that standard deviation is $10 \%$, or less, of the mean. Perform additional replicates as needed to meet the criteria.

13. Repeat steps $3.4-3.12$ for each additional nozzle, pressure, nozzle orientation and airspeed combination of interest.

14. Export and save droplet size data using the method provided within the operating software.

\section{Representative Results}

The resulting data from this method can be expressed in a variety of formats, depending on the user's preference and the operational capabilities of the laser diffraction system. Typically this data is presented as a plot of the volume weighted droplet size distribution (Figures $\mathbf{1}$ and $\mathbf{2}$ ) or as descriptive droplet size metrics (Tables 1 and 2). These results can then be used to examine the impact that changes in nozzle or operational parameters have on the resulting spray droplet size. 
We examined two different aerial spray nozzles, both with the same orifice size but with different spray fan angles. With these two aerial nozzles, we also examined the effects of spray pressure and airspeed on droplet size. Examining the 2015 nozzle operated at a spray pressure of $207 \mathrm{kPa}$ and comparing the volume weighted distributions resulting from the same nozzle being operated in $53.6 \mathrm{~m} / \mathrm{sec}$ versus $71.5 \mathrm{~m} / \mathrm{sec}$ airspeed, it is immediately obvious that the higher airspeeds results in a dramatic shift in the incremental and cumulative distributions toward smaller droplet diameters (Figures 1 and 2) which is the result of increased breakup of spray droplets at the higher airspeed. While the graphical representation of the results provide a very visual representation of the results, quantitative values derived from these distributions are more practical for larger data sets. Typical droplet size metrics used in agricultural spray research include the $D_{\mathrm{v} 0.1}, D_{\mathrm{v} 0.5}$ and $D_{\mathrm{v} 0.9}$ values, which correspond to the droplet diameters such that 10,50 and $90 \%$ (respectively) of the spray volume is contained in droplets of equal or lesser diameter. These data are the same as those shown in the graphical distributions, but provide a more convenient format of expressing the data. Comparing the data for both the 2015 and 4015 spray nozzles at both pressures and all three airspeeds, general trends can be observed (Table 1). The 4015 flat fan nozzle results in smaller droplet sizes than the 2015 at the same pressure and airspeed, as indicated by the smaller volume weighted diameters $\left(D_{\vee_{0} .1}, D_{v_{0.5}}\right.$, and $\left.D_{v_{0} .9}\right)$ and the increase in the total volume of the spray comprised of droplet of $100 \mu m$ or less. $D_{v_{0.1}}, D_{v_{0.5}}$, and $\mathrm{D}_{\mathrm{v} 0.9}$ are the droplet diameters such that 10,50 and $90 \%$, respectively, of the total spray volume is comprised of droplets of equal or lesser diameter. This is the result of the increase spray fan angle seeing greater breakup at the outer edges of the liquid fan angle. Within the same nozzle type and spray pressure, all droplet size metrics decrease with increasing airspeeds, again as a result of increasing breakup of droplets at the higher airspeeds. An interesting phenomenon with the aerial spray nozzles is seen when looking at the effects of spray pressure within each nozzle and airspeed combination. All else remaining equal, as pressure increases, so does droplet size ${ }^{11}$. This is caused by a decrease in the relative velocity difference between the liquid exiting the nozzle and the surrounding airstream, as the liquid exit velocity increases as pressure increases (Table 1$)^{13}$.

Looking at the results from the ground nozzles and spray pressures tested, the effect of nozzle type on droplet size is significant with the TTI11003 resulting in droplet sizes that are more than double that the XRC11003 and the Al11003 droplet sizes falling in the middle of the other two (Table 2). Within each nozzle type, the effects of pressure can be observed with droplet sizes decreasing with increased spray pressure.

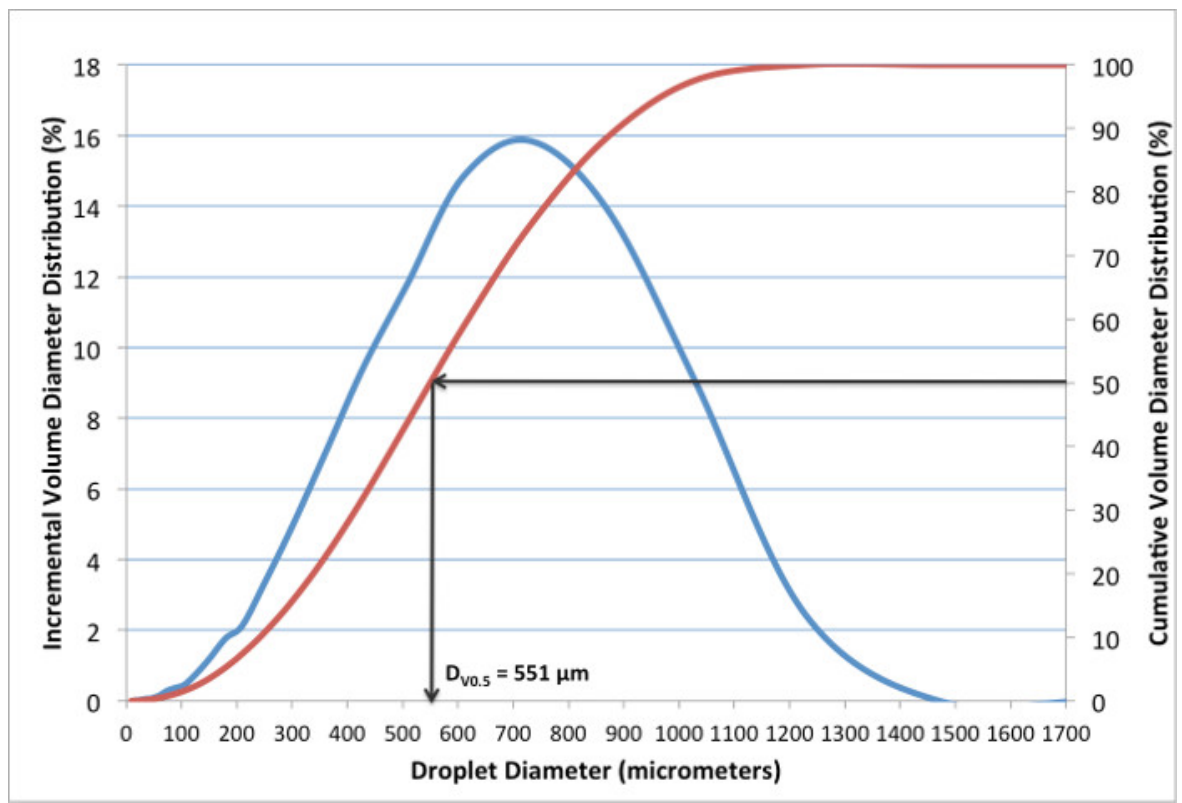

Figure 1. Incremental droplet size distribution for a 20 degree flat fan aerial spray nozzle with a \#15 orifice operated at $207 \mathrm{kPa}$ and in an airspeed of $53.6 \mathrm{~m} / \mathrm{sec}$. The blue curve represents the incremental volume weighted distribution which provides the percentage of the total spray volume contained in droplets falling with the range of each measurement bin as measured by the laser diffraction system. The red curve is the same data, but represented as cumulative data. The cumulative data allows for the volume-weighted diameters specific to a certain percentage of total spray volume to be determined. As illustrated in the figure, to obtained the $\mathrm{D}_{\mathrm{v} 0.5}$ volume diameter, locating the $50 \%$ point on the cumulative curve and the associated droplet diameter shows that $50 \%$ of the total spray volume is contained in spray droplets of diameter $551 \mu \mathrm{m}$ or smaller. Please click here to view a larger version of this figure. 


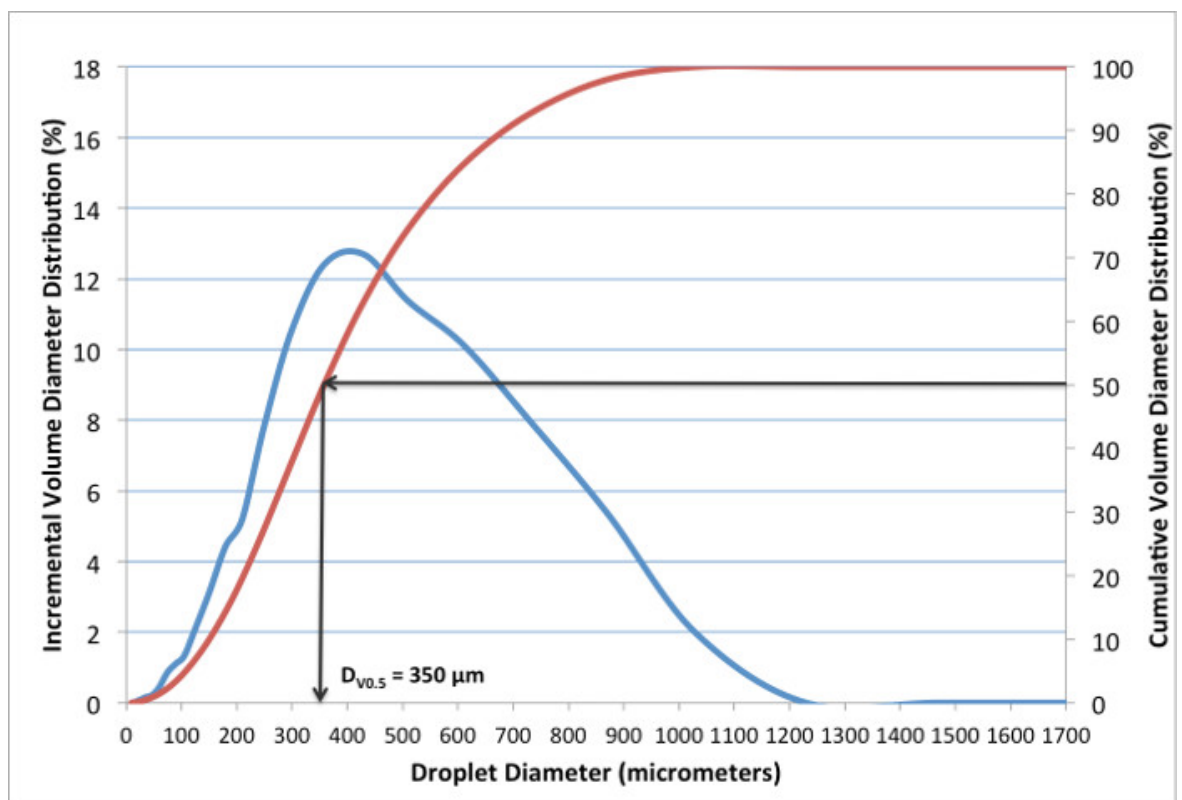

Figure 2. Incremental droplet size distribution for a $\mathbf{4 0}$ degree flat fan aerial spray nozzle with a \#15 orifice operated at $207 \mathrm{kPa}$ and in an airspeed of $71.5 \mathrm{~m} / \mathrm{sec}$. As in Figure 1, the blue curve represents the incremental volume weighted distribution and the red curve is the cumulative distribution. Compared to the results shown in Figure 1, the incremental distribution shows a significant shift toward smaller droplet diameters as a result of the increased airspeed and therefore secondary droplet breakup. Determining the $\mathrm{D}_{\mathrm{V} 0.5}$ volume diameter shows that $50 \%$ of this spray volume is contained in droplets of diameter $350 \mu \mathrm{m}$ or smaller. Please click here to view a larger version of this figure.

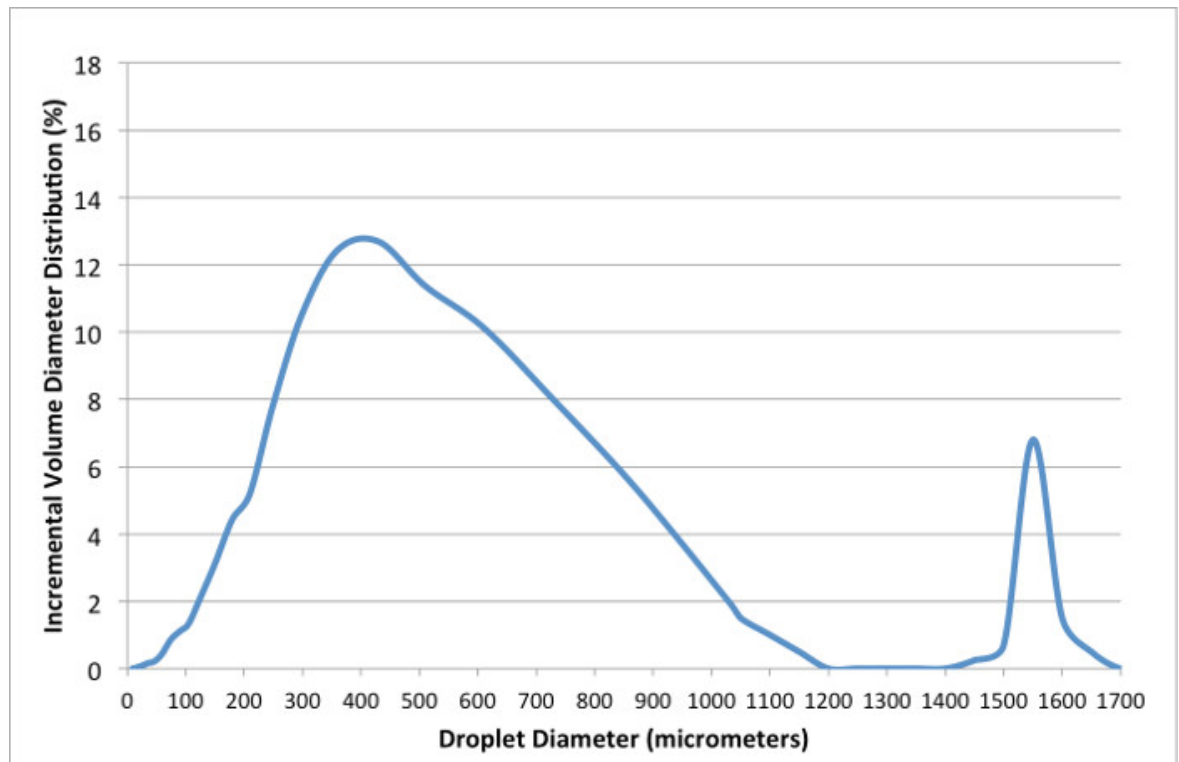

Figure 3. Incremental droplet size distribution with false peak example plot. The secondary, smaller peak on the right, toward the larger end of the droplet size scale is typically the result of either vibrations or other noise in the system or the presence of ligaments associated with incomplete atomization within the spray cloud. As droplet size distributions for typical agricultural spray nozzles and solutions are typically lognormally distributed, the presence of a secondary peak in the distribution may be a valid result from an atypical spray solution and/or nozzle combination, but is more likely an indicator of some confounding issue in the measurement process. Please click here to view a larger version of this figure. 


\begin{tabular}{|c|c|c|c|c|c|c|}
\hline \multirow[t]{2}{*}{ Nozzle } & \multirow[t]{2}{*}{ Pressure (kPa) } & \multirow[t]{2}{*}{ Airspeed (m/sec) } & \multicolumn{3}{|c|}{ Volume Weighted Diameters $(\mu \mathrm{m})$ [Mean \pm St. Dev.] } & \multirow{2}{*}{$\begin{array}{l}\text { Percent Spray } \\
\text { Volume Less than } \\
100 \mu \mathrm{m}\end{array}$} \\
\hline & & & $\mathrm{D}_{\mathrm{V} 0.1}$ & $D_{\mathrm{V} 0.5}$ & $\mathrm{D}_{\mathrm{V} 0.9}$ & \\
\hline \multirow[t]{6}{*}{2015} & \multirow[t]{3}{*}{207} & 53.6 & $243.5 \pm 2.5$ & $551.8 \pm 4.6$ & $903.0 \pm 25.4$ & $1.4 \pm 0.05$ \\
\hline & & 62.6 & $192.1 \pm 0.5$ & $444.5 \pm 1.5$ & $781.7 \pm 7.0$ & $2.4 \pm 0.04$ \\
\hline & & 71.5 & $147.0 \pm 2.8$ & $350.6 \pm 6.1$ & $673.3 \pm 14.6$ & $4.5 \pm 0.18$ \\
\hline & \multirow[t]{3}{*}{414} & 53.6 & $289.1 \pm 3.1$ & $655.6 \pm 2.1$ & $1208.7 \pm 11.6$ & $0.8 \pm 0.03$ \\
\hline & & 62.6 & $237.6 \pm 0.1$ & $542.7 \pm 1.7$ & $1072.5 \pm 13.7$ & $1.3 \pm 0.01$ \\
\hline & & 71.5 & $170.8 \pm 1.1$ & $400.6 \pm 3.3$ & $732.1 \pm 6.4$ & $3.2 \pm 0.05$ \\
\hline \multirow[t]{6}{*}{4015} & \multirow[t]{3}{*}{207} & 53.6 & $230.2 \pm 1.3$ & $514.9 \pm 1.9$ & $863.3 \pm 1.2$ & $1.5 \pm 0.03$ \\
\hline & & 62.6 & $175.1 \pm 2.0$ & $404.5 \pm 2.6$ & $714.2 \pm 3.0$ & $3.1 \pm 0.10$ \\
\hline & & 71.5 & $146.6 \pm 0.8$ & $344.5 \pm 2.4$ & $656.4 \pm 9.5$ & $4.6 \pm 0.05$ \\
\hline & \multirow[t]{3}{*}{414} & 53.6 & $255.2 \pm 2.4$ & $557.3 \pm 2.3$ & $994.9 \pm 8.1$ & $1 \pm 0.04$ \\
\hline & & 62.6 & $200.1 \pm 2.6$ & $449.4 \pm 7.0$ & $774.9 \pm 10.7$ & $2.1 \pm 0.06$ \\
\hline & & 71.5 & $165.5 \pm 1.4$ & $383.5 \pm 2.6$ & $696.8 \pm 4.9$ & $3.4 \pm 0.08$ \\
\hline
\end{tabular}

Table 1. Volume weighted diameters (averages \pm standard deviations across three replicate measurements) for 2015 and 4015 flat fan aerial spray nozzles operated at spray pressures of 207 and $414 \mathrm{kPa}$ and in airspeeds of $53.6,62.6$ and $71.5 \mathrm{~m} / \mathrm{sec}$.

\begin{tabular}{|c|c|c|c|c|c|}
\hline \multirow[t]{2}{*}{ Nozzle } & \multirow[t]{2}{*}{ Pressure (kPa) } & \multicolumn{3}{|c|}{ Volume Weighted Diameters $(\mu \mathrm{m})$ [Mean \pm St. Dev.] } & \multirow{2}{*}{$\begin{array}{l}\text { Percent Spray } \\
\text { Volume Less than } \\
100 \mu \mathrm{m}\end{array}$} \\
\hline & & $\mathrm{D}_{\mathrm{V} 0.1}$ & $\mathrm{D}_{\mathrm{V} 0.5}$ & $\mathrm{D}_{\mathrm{V} 0.9}$ & \\
\hline \multirow[t]{2}{*}{ XRC11005 } & 276 & $115.1 \pm 2.1$ & $268.2 \pm 5.6$ & $451.0 \pm 18.0$ & $7.2 \pm 0.28$ \\
\hline & 414 & $101.0 \pm 0.0$ & $244.2 \pm 0.7$ & $424.3 \pm 4.3$ & $9.8 \pm 0.01$ \\
\hline \multirow[t]{2}{*}{ Al11005 } & 276 & $227.6 \pm 1.9$ & $468.9 \pm 4.1$ & $763.0 \pm 22.0$ & $1.1 \pm 0.03$ \\
\hline & 414 & $183.4 \pm 0.6$ & $399.6 \pm 0.9$ & $668.6 \pm 2.5$ & $2.2 \pm 0.05$ \\
\hline \multirow[t]{2}{*}{ TTI11005 } & 276 & $365.3 \pm 5.3$ & $711.9 \pm 16.9$ & $1013.8 \pm 26.1$ & $0.1 \pm 0.00$ \\
\hline & 414 & $311.5 \pm 4.0$ & $645.7 \pm 12.3$ & $992.7 \pm 24.7$ & $0.2 \pm 0.01$ \\
\hline
\end{tabular}

Table 2. Volume weighted diameters (averages \pm standard deviations across three replicate measurements) for three ground sprayer nozzles (XRC11005, Al11005 and TTI11005) operated at spray pressures of 276 and $414 \mathrm{kPa}$.

\section{Discussion}

There are a number of critical steps that should be followed when applying this method. With both aerial and ground nozzle evaluations, the distance from the exit of the nozzle to the line of measurement should be verified prior to any measurement. Any variance in this distance can have a significant impact on the results. Similarly, the concurrent airspeed used in ground nozzle testing should be verified and adjusted to the $6.7 \mathrm{~m} / \mathrm{sec}$ recommended. Differences in airspeed from that recommended will significantly influence the results due to sampling bias issues at lower airspeeds, and potentially increase secondary breakup at higher airspeeds. Also, proper alignment of the laser diffraction system components is critical in order to ensure the system is operating at the accuracy and precision specification certified by the manufacturer. Proper setup and alignment of the nozzles relative to the concurrent airflow is critical to ensuring quality data, as even slight misalignments of a few degrees in the nozzles positioning can result in significant impacts on the resulting droplet size data.

The methods presented can be applied to any spray nozzle configuration or spray solution for both ground and aerial system. With ground sprayers, changes in spray droplet size are typically a function of nozzle type and size, spray pressure and spray solution type. With aerial sprayer the additional role of changes in airspeed and the orientation of the nozzle to surrounding airstream are critical to the resulting droplet size. This method can be used to evaluate the combined effect of these factors on the final droplet size. However there are rare instances when some modifications to the recommended methods are required. Specifically, spray solutions or nozzles that require further distances from the nozzle for complete breakup of spray into discrete particles will require adjusting the distance between nozzle and measurement point. To date, the only nozzle/spray solution treatments that have required this sort of adjustment have been straight stream nozzles at all operational settings and narrow angle flat fan nozzles with spray additives that increase the solutions viscosity, when measured under aerial application testing conditions. The laser diffraction system will still return droplet size data in the event of incomplete breakup of the spray cloud, but the resulting data will typically be biased toward much larger droplet sizes as a result of spray ligaments being measured by the system. While these ligaments are not readily apparent to the naked eye, their presence will typically show up visually in the distribution plot as a secondary peak at the larger end of the droplet size scale (Figure 3). Though caution is advised in assuming that this secondary peak is the result of the presence of ligaments, as external vibrations or other interference with the laser diffraction system may cause a similar response. As a user's experience level increases, making the distinction between the two based on errors becomes easier. In the case where spray atomization is incomplete, we have found that extending the sampling distance to $1.8 \mathrm{~m}$ (for aerial spray nozzles) resolves the issue and returns quality data. This 1.8 $\mathrm{m}$ distance is in fact the standard distance at which our group evaluates all straight stream nozzles under aerial application conditions. When 
working with ground sprayer nozzles, there are a class of nozzle designs that use a twin, flat fan orifice outlet the may require modification to the nozzle mounting setup to insure the entire spray plume passes through the sampling area without fouling the laser diffraction system's lenses.

While this method is designed to minimize the sampling bias due to spatial biases associated with laser diffraction systems, it does not completely eliminate them, meaning that the droplet size values return cannot be taken as "absolute". Laser diffraction does not provide a means to measure, and adjust, the resulting droplet size data for the non-homogeneous droplet velocities amongst the different droplet sizes in the composite spray cloud. This becomes critical when inter-laboratory data sets are compared, particularly with respect to ground spray nozzles. The method currently accepted to standardize the results and allow comparisons between laboratories uses a series of highly calibrated reference spray nozzles, whose droplet size data are used to establish a set of classification categories. Evaluation of these nozzles should be conducted as part of every droplet sizing evaluation. Further details on the nozzles and classification definitions can be found in the American Society of Agricultural and Biological Engineers (ASABE) "Spray Nozzle Classification by Droplet Spectra" International Standard (ASAE/ANSI, 2009).

As discussed in the Introduction, there are other droplet sizing systems besides laser diffraction. Where laser diffraction provides a composite measure of droplet size across the entire spray plume, these other methods focus in on a small area with the spray cloud, sampling only a small portion of the overall spray cloud. Obtaining a representative sample of the entire plume with these others methods requires a much more rigorous, and time consuming, multi-chordal traverse of the spray plume's cross section area, resulting in a large number of sub-samples that must be combined to generate a composite result. This requires significantly more time than using laser diffraction.

Once this method has been successfully integrated into a research program and the techniques mastered by the users, the next challenge is conducting well-structured experiments aimed at understanding the role each of the influence factors play with respect to the formation of droplet size. This is a bigger challenge than it seems given the seemingly endless combination of nozzle type, nozzle setup and operational factors, airspeed and nozzle position (aerial spraying) and real-world tank mixes used by the agricultural application industry. Even more of a challenge is finding a way the makes this information available to the applicators in a format that is easily useable. One option our group has used with great success is a class of experimental designs called response surfaces that allow for the development of droplet size prediction models based on a limited number of experimental treatments allowing for an extremely efficient evaluation of multiple spray nozzles and solutions ${ }^{14,15}$. This structured design method has been used to develop a series of droplet size models for the most commonly used aerial ${ }^{11}$ and ground nozzles ${ }^{16}$ used by agricultural applicators.

\section{Disclosures}

The authors have nothing to disclose.

\section{Acknowledgements}

This study was supported in part by a grant from the Deployed War-Fighter Protection (DWFP) Research Program, funded by the U.S. Department of Defense through the Armed Forces Pest Management Board (AFPMB). Mention of trade names or commercial products in this publication is solely for the purpose of providing specific information and does not imply recommendation or endorsement by the U.S. Department of Agriculture. USDA is an equal opportunity employer.

\section{References}

1. Bouse, L. F. Effect of nozzle type and operation on spray droplet size. Trans. ASAE. 37 (5), 1389-1400. (1994).

2. Hewitt, A. Droplet size and agricultural spraying, Part I: Atomization, spray transport, deposition, drift and droplet size measurement techniques. Atomization Spray. 7 (3), 235-244 (1997).

3. Black, D.L., McQuay, M.Q., and Bonin, M.P. Laser-based techniques for particle-size measurement: A review of sizing methods and their industrial applications. Prog. Energy Combust. Sci. 22 (3), 267-306. (1996).

4. SDTF Spray Drift Task Force Study No. A95-010, Miscellaneous Nozzle Study. EPA MRID, No. 44310401 (1997).

5. Dodge, L. G. Comparison of performance of drop-sizing instruments. Appl. Optics. 26 (7), 1328-1341 (1987).

6. Arnold, A. C. A comparative study of drop sizing equipment for agricultural fan-spray atomizers. Aeronaut. Sci. Tech., 12 (2), $431-445$ (1990).

7. Teske, M.E., Thistle, H.W., Hewitt, A.J. and Kirk, I.W. Conversion of droplet size distributions from PMS optical array probe to Malvern laser diffraction. Atomization Spray. 12 (1-3), 267-281 (2002).

8. Fritz, B.K., et al. Measuring droplet size of agricultural spray nozzles - Measurement distance and airspeed effects. Atomization Spray 24 (9), 747-760 (2014).

9. ANSI/ASAE S572.1 Spray Nozzle Classification by Droplet Spectra, American Society of Agricultural Engineers. St. Joseph, MI. 4, pp. 1-3 (2009).

10. Fritz, B.K., et al. Comparison of drop size data from ground and aerial nozzles at three testing laboratories. Atomization Spray 24 (2), 181-192 (2014).

11. Fritz, B.K., Hoffmann, W.C. Update to the USDA-ARS fixed-wing spray nozzle models. Trans ASABE. 58 (2), $281-295$ (2015).

12. Sympatec Inc. HELOS Central Unit Operating Instructions. Sympatec GmbH, Clausthal-Zellerfeld, Germany (2002).

13. Elbanna, H., Rashed, M.I., Ghazi, M.A. Droplets from liquid sheets in an airstream. Trans ASAE. 27 (3), 677-679 (1984).

14. Box, G.E.P., Behnken, D.W. Some new three-level designs for the study of quantitative variables. Technometrics. 2(4), 455-475 (1960).

15. Myers, R.H., Montgomery, D.C., Anderson-Cook, C.M. Response Surface Methodology: Process and Product Optimization Using Designed Experiments, 3rd Edition. Wiley Press, 704 p. ISBN: 978-0-470-17446-3, (2009).

16. Fritz, B.K., Hoffmann, W.C., Anderson, J., Response surface method for evaluation of the performance of agricultural application spray nozzles. Pesticide Formulation and Delivery Systems: 35th Volume, ASTM STP1587. G. R. Goss, Ed., ASTM International, West Conshohocken, PA, pp. 61-76 (2016). 